\title{
Chromospheric activity and lithium line variations in the spectra of the spotted star LQ Hydrae ${ }^{\star}, \star$
}

\author{
M. Flores Soriano, K. G. Strassmeier, and M. Weber
}

\author{
Leibniz-Institut für Astrophysik Potsdam (AIP), An der Sternwarte 16, 14482 Potsdam, Germany \\ e-mail: [mflores;kstrassmeier;mweber]@aip.de
}

Received 16 October 2014 / Accepted 12 January 2015

\begin{abstract}
Context. Although the relationship between lithium abundance in stars and their magnetic activity is commonly accepted, it is still unclear how the different phenomena related to it can increase the amount of $\mathrm{Li}$, reduce its depletion, or be a source of bias for the measurements.

Aims. We study the rotational modulation of chromospheric and photospheric parameters of the young, active, single K2 dwarf LQ Hya and their connection with the variability of the Li I 6708 A line.

Methods. A total of 199 high-resolution STELLA spectra and quasi-simultaneous photometry were used to compute effective temperature, gravity, and chromospheric activity indicators such as $\mathrm{H} \alpha$ and $\mathrm{H} \beta$ emission, Balmer decrement, and chromospheric electron density, as a function of the rotational phase. The variation of the Li I $6708 \AA$ line was characterized in terms of equivalent width, abundance, and of ${ }^{6} \mathrm{Li} /{ }^{7} \mathrm{Li}$ isotopic ratio in the form of line shifts.

Results. Photospheric and chromospheric parameters show clear rotational modulation. Effective temperatures and continuum variations reveal a higher concentration of cool spots on the side of the star on which we also detect stronger chromospheric activity. Increased electron densities and the modulation of the $\mathrm{He} \mathrm{I}_{3}$ line suggest that the source of this activity can be a combination of plages and repeated low-intensity flares. The Li line and other temperature-sensitive lines are clearly enhanced by the spots located on the most active side of the star. Li abundances calculated taking into account the temperature variations simultaneously show, although with high dispersion, a small overabundance of this element that correlates well with the surface magnetic activity. In addition, the $\mathrm{Li}$ line center is more intensely redshifted than in the other hemisphere, which might be interpreted as a weak enrichment of ${ }^{6} \mathrm{Li}$.
\end{abstract}

Key words. stars: abundances - stars: activity - stars: chromospheres - stars: flare - stars: rotation - stars: individual: LQ Hydrae

\section{Introduction}

Lithium atoms are systematically destroyed by thermonuclear reactions in the interior of late-type stars as convection transports material from the surface layers to hotter regions (Iben 1967). The progressive depletion of lithium is therefore considered a useful tool for studying mixing processes in stellar interiors and an excellent age indicator. However, the large spread of Li abundances found among $\mathrm{G}$ and $\mathrm{K}$ dwarfs in young open clusters (see, e.g., Duncan \& Jones 1983; Soderblom et al. 1993), and the detection of high amounts of $\mathrm{Li}$ in evolved stars (see, among others, Wallerstein \& Sneden 1982; Brown et al. 1989), have shown that the connection between stellar age and Li abundance is not as simple as initially thought.

Although the spread of the Li abundance is expected to be the result of a complex interplay, phenomena related to the stellar magnetic activity are generally acknowledged to play a relevant role. Active binary components are particularly good mixing

* Based on data obtained with the STELLA robotic telescope in Tenerife, an AIP facility jointly operated by AIP and IAC, and the Vienna-Potsdam Automatic Photoelectric Telescopes at Fairborn Observatory in Arizona, operated by AIP.

$\star \star$ The numerical results of the spectral analysis are only available at the CDS via anonymous ftp to

cdsarc.u-strasbg.fr (130.79.128.5) or via

http://cdsarc.u-strasbg.fr/viz-bin/qcat?]/A+A/575/A57 tracers because they rotate faster than single stars. A trend of their $\mathrm{Li}$ abundance as a function of rotation period was observed just recently (Strassmeier et al. 2012), but with a dispersion of as much as three to four orders of magnitude. On the basis of the Li I 6708 A line enhancement in sunspots (Grevesse 1968; Traub \& Roesler 1971; Giampapa 1984), Giampapa (1984) suggested that cool starspots might introduce ambiguities into abundance determinations and contribute significantly to the scatter. Fekel (1996) and Cutispoto (2002) reviewed the correlation between $\mathrm{Li}$ equivalent width $(\mathrm{EW})$ variations and starspots, concluding that the conditions for producing significant EW variations are rarely met and that surface inhomogeneities can only explain a small fraction of the observed Li abundance spread. The effects of magnetic fields on the convective envelope of pre-main sequence stars were modeled by Ventura et al. (1998), who found that even relatively low values can inhibit convection and thus significantly reduce the Li depletion. On the other hand, the stellar model constructed by Li et al. (2014) shows that magnetic instabilities might produce additional mixing during the pre-main sequence, resulting in strong depletion. An anisotropic supergranulation pattern was found on the surface of the super Li-rich but magnetically non-active giant HD 233517 by means of Doppler imaging (Strassmeier et al. 2015). It favors an additional mixing scenario as the cause for its extreme surface $\mathrm{Li}$ abundance opposite to an engulfing event. Another way to influence the amount of lithium is the production of fresh atoms 
by nuclear reactions during flares (see, e.g., Fowler et al. 1955; Canal 1974; Canal et al. 1975; Ramaty et al. 2000; Tatischeff $\&$ Thibaud 2007). This type of event has been proposed as a possible explanation for the high $\mathrm{Li}$ abundance observed in some stars (Pallavicini et al. 1992; Deliyannis \& Malaney 1995; Mathioudakis et al. 1995; Favata et al. 1996). Nevertheless, the Li I $6708 \AA$ line enhancement observed during a long-duration flare in an active binary by Montes \& Ramsey (1998) is still the only direct detection.

Stars with an inhomogeneous distribution of active regions allow studying the influence of the magnetic activity on the lithium line as the star rotates. One of these stars is the young, fast rotating K2 dwarf LQ Hya (HD 82558). It was recognized as a chromospherically active star by Bidelman (1981) and Heintz (1981) because of the strong Ca II H\&K emission. Fekel et al. (1986b) observed that the $\mathrm{H} \alpha$ line was completely filled by emission and classified the star as a BY Draconis variable. From its fast rotation, high lithium abundance, and chromospheric activity, Fekel et al. (1986a) concluded that LQ Hya is a single star "at least as young as the youngest Pleiades star". Strassmeier et al. (1993) and Frasca et al. (2008; hereafter Papers I and II respectively), and more recently Cao \& Gu (2014), computed chromospheric parameters as a function of the rotational phase. All of them found higher chromospheric emission in regions associated with starspots. Strong flares have been detected in LQ Hya in wavelengths from the optical (Montes et al. 1999) to X-rays (Covino et al. 2001), including UV (Ambruster \& Fekel 1990; Montes et al. 1999). Frequent low-level flare activity has been also reported by Saar \& Bookbinder (1998).

The aim of the present work is to analyze the rotational modulation of different magnetic activity indicators with superior spectral time coverage and their connection with the variability of the Li I $6708 \AA$ line. In particular, we are interested in determining wheter an inhomogeneous distribution of regularly occurring low-intensity flares can produce a local overabundance of lithium. In Sect. 2 we describe the observations. The chromospheric activity indicators and their sources of emission are analyzed in Sect. 3. In Sect. 4 we characterized the behavior of the $\mathrm{Li}$ I $6708 \AA$ line at different rotational phases and correlate it with the rotational modulation of the chromospheric activity. Finally, Sect. 5 summarizes and concludes the paper.

\section{Observations and data reduction}

\subsection{STELLA/SES spectroscopy}

A time series of 199 echelle spectra was taken with the STELLA Echelle Spectrograph (SES) at the robotic $1.2 \mathrm{~m}$ STELLA-I telescope at the Observatorio del Teide in Tenerife, Spain (Strassmeier et al. 2010). Observations spanned the period between December 2011 and April 2012. The observing $\log$ and the numerical results obtained from the spectra can be found at the CDS. The integration time was set to $3600 \mathrm{~s}$, providing signal-to-noise ratios $(\mathrm{S} / \mathrm{N})$ between 40 and 120 depending on the actual weather conditions. The spectra cover the wavelength range from $4760 \AA$ to $7640 \AA$ with a resolving power of $R=30000$, corresponding to a spectral resolution of $0.21 \AA$ at $6400 \AA$ and to a dispersion of $0.04 \AA /$ pix.

The SES is a fiber-fed white-pupil echelle spectrograph with a fixed wavelength format of normally 388-882 nm and $R=55000$. During our observation period, we interrupted the ongoing spectrograph upgrade to a new cross disperser, a new optical camera, and a larger CCD. However, the collimator was slightly misaligned and the old spectrograph camera out of focus, which caused a significantly lower effective spectral resolution than nominal. The instrument is located in a dedicated room on a stabilized optical bench and is fed by a $12 \mathrm{~m}$ long $50 \mu \mathrm{m}$ Ceram-Optec fibre, which enables it to deliver radial velocities on a $30 \mathrm{~m} \mathrm{~s}^{-1} \mathrm{rms}$ level. The CCD was still the e2v 42-40 2048×2048 $13.5 \mu \mathrm{m}$-pixel device.

The SES spectra are automatically reduced using the IRAF ${ }^{1}$ based STELLA data-reduction pipeline (Weber et al. 2008). The images were corrected for bad pixels and cosmic-ray impacts. Bias levels were removed by subtracting the average overscan from each image followed by the subtraction of the mean of the (already overscan-subtracted) master bias frame. The target spectra were flattened by dividing by a nightly master flat that had been normalized to unity. The nightly master flat itself is constructed from around 50 individual flats observed during dusk, dawn, and around midnight. After removal of the scattered light, the one-dimensional spectra were extracted with the standard IRAF optimal extraction routine. Then they were wavelength calibrated using consecutively recorded Th-Ar spectra. A flux normalized synthetic spectrum was used to fit the blaze function to the target spectra. The observed spectra were finally divided by this blaze function.

Radial velocities were determined from a simultaneous cross-correlation of 40 echelle orders with a synthetic spectrum matching the target properties of LQ Hya (see Weber \& Strassmeier 2011 for a detailed description). Numerous radial velocity standards and stellar comparison targets were also observed with the same setup.

Fifty selected spectral orders were used to determine the stellar effective temperature, gravity, line broadening, and metallicity. They basically include all orders except for those with many telluric lines and $\mathrm{H} \alpha$. Our numerical tool PARSES ("PARameters from SES"; Allende Prieto 2004; Jovanovic et al. 2013) is implemented as a suite of Fortran programs in the STELLA data analysis pipeline. It is based on the synthetic spectrum-fitting procedure described in Allende Prieto et al. (2006). All calculations were made using MARCS model atmospheres (Gustafsson et al. 2008) with the VALD3 line list (Kupka et al. 2011; and updates on some specific $\log g f$ values). Microturbulence and macroturbulence were fixed to $1.1 \mathrm{~km} \mathrm{~s}^{-1}$ and $2.5 \mathrm{~km} \mathrm{~s}^{-1}$. This grid was then used to compare the model spectra with the fifty selected echelle orders of each spectrum. We verified this approach by applying it to the ELODIE library (Prugniel \& Soubiran 2001) and used linear regressions to the offsets with respect to the literature values to place our PARSES values on the same zero point. The program did not give satisfactory stellar parameters for 17 of the 199 spectra. For these we estimated the values using nearest-neighbors interpolation in phase and time. The uncertainties were calculated as the local standard deviation of all PARSES measurements within a window of 0.1 phases centered at the point under consideration.

The rotational modulation of radial velocities and effective temperatures can be seen in the top and middle panel of Fig. 1, respectively. As all other observations in this paper, they were phased with the ephemeris HJD $=2455919.67+1.60066 \cdot E$, where the zero epoch is arbitrary and the rotation period of the star is from Kővári et al. (2004).

\subsection{Amadeus APT photometry}

Continuous phase-resolved photometry was obtained with one of the two Vienna-Potsdam $0.75 \mathrm{~m}$ twin automatic photoelectric

1 IRAF is hosted by the National Optical Astronomy Observatories in Tucson, Arizona at URL iraf. noao. edu. 
M. Flores Soriano et al.: Chromospheric activity and lithium line variations in the spectra of the spotted star LQ Hydrae

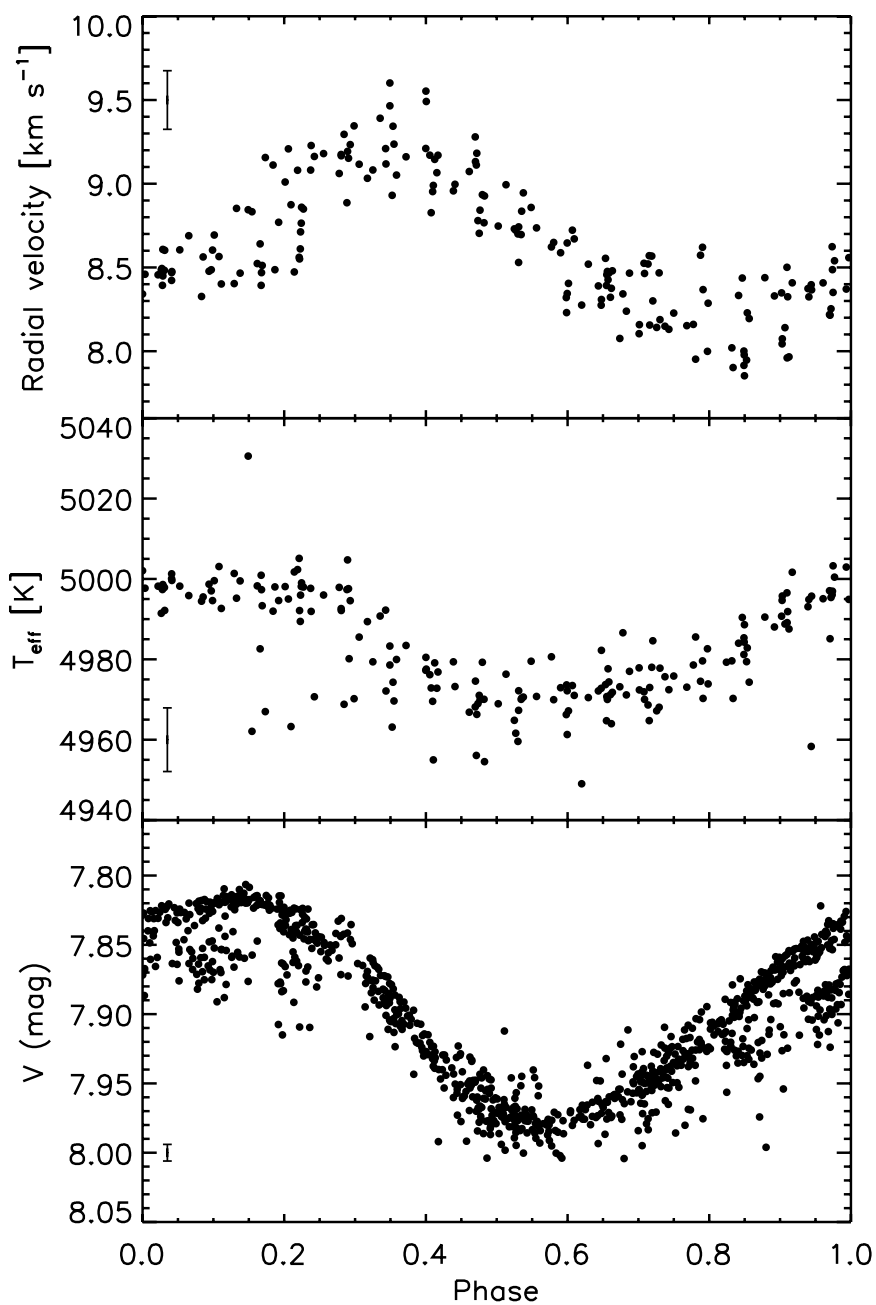

Fig. 1. Top panel: radial velocity of LQ Hya from cross correlation with a synthetic template. Because LQ Hya is a single star, its rotational modulation is a consequence of the asymmetric distribution of spots. Middle panel: effective temperature of LQ Hya calculated for each spectrum with the synthetic spectrum-fitting tool PARSES. Bottom panel: rotational modulation of the $V$-band photometry contemporaneous to the spectra. Average error bars are given at the left side of each panel.

telescopes (APTs) at Fairborn Observatory in southern Arizona (Strassmeier et al. 1997). The Potsdam-APT Amadeus (T7) was used quasi-simultaneously with the STELLA observations. It achieved an external precision of $4-6 \mathrm{mmag}$ in JohnsonCousins $V$. A total of $965 V$ data points were obtained and are presented in this paper in Fig. 1. The integration time was $10 \mathrm{~s}$. All measurements were made differentially with respect to HD 82447 and HD 82508 as the comparison and check star, respectively. Both stars appear constant to within the telescope's internal precision (Rice \& Strassmeier 1998). The APT data were transformed to absolute values with nightly observations of up to 60 standard stars. For further details of the observing procedure and the APT data reduction in general, we refer to Granzer et al. (2001).

\section{Chromospheric activity}

\section{1. $\mathrm{H} \alpha$ and $\mathrm{H} \beta$ emission}

In a similar way as reported by Frasca et al. (2011) for the young star KIC 8429280, we evaluated the chromospheric activity of LQ Hya from the emission of the Balmer $\mathrm{H} \alpha$ and $\mathrm{H} \beta$ lines. We

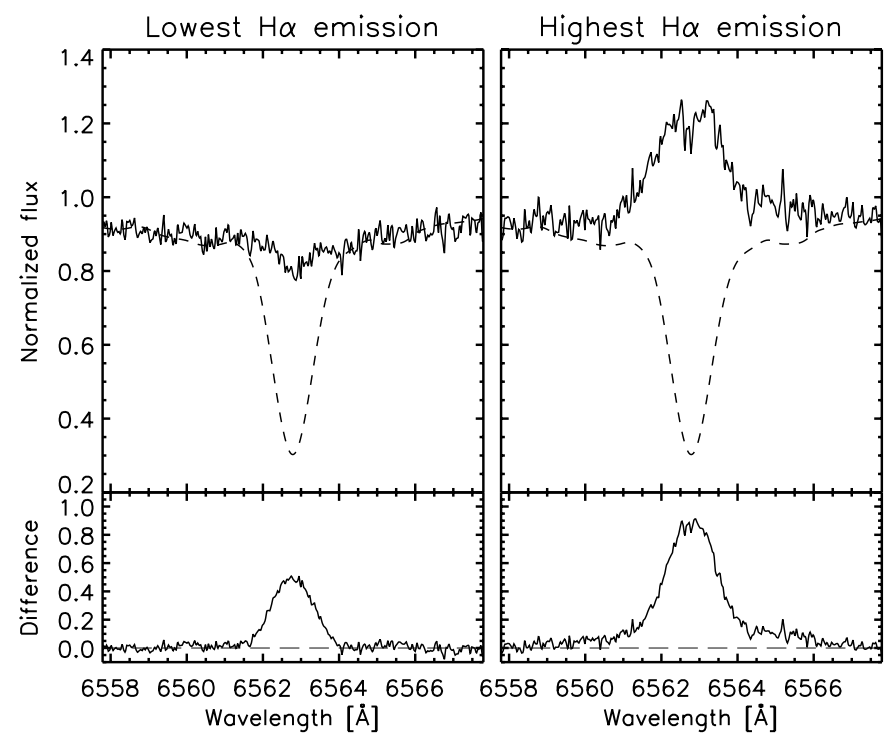

Fig. 2. Upper panels: $\mathrm{H} \alpha$ line during lowest (left) and highest emission (right). The non-active reference star (HD 3765) is shown as a dashed line. Bottom panels: difference between LQ Hya and referencestar spectrum.

applied the so-called spectral synthesis method (see, e.g., Herbig 1985; Frasca \& Catalano 1994), which provides the net emission of the lines by subtracting a spectrum of a non-active star of similar classification.

As in Paper II, we used as non-active template a highresolution $(R=42000)$ spectrum of HD 3765 from the ELODIE database. We selected this star because it has a much lower activity level than LQ Hya, but the same spectral type and a similar metallicity. To enable the direct comparison with the observed spectra, the template was convolved with a Gaussian of $F W H M=\lambda \sqrt{R_{\mathrm{STELLA}}^{-2}-R_{\mathrm{ELODIE}}^{-2}}$ to reduce its resolution to $R=30000$, and subsequently broadened to mach the rotational profile of LQ Hya.

The equivalent widths of the net $\mathrm{H} \alpha$ and $\mathrm{H} \beta$ emission profiles were evaluated by integrating each one of the 199 spectra resulting from the subtraction of the template. Their corresponding errors, as for all other equivalent widths in this paper, were calculated with the expression resulting from the propagation of the uncertainties

$\sigma_{E W}=\sigma\left(F_{\text {STELLA }}\right) \sqrt{\Delta \lambda \cdot \mathrm{d} \lambda_{\text {STELLA }}}+\sigma\left(F_{\text {ELODIE }}\right) \sqrt{\Delta \lambda \cdot \mathrm{d} \lambda_{\text {ELODIE }}}$,

where $\sigma(F)$ is the standard deviation of the spectrum measured in regions without lines, $\Delta \lambda$ is the size of the integration window, and $\mathrm{d} \lambda$ is the pixel sampling.

We observe that both Balmer lines are strongly variable. During the lowest emission, $\mathrm{H} \alpha$ is shown as a line in weak absorption with its core immediately below the continuum level, while it is clearly in emission during the maximum (see Fig. 2). Paper II reported on a weaker variability in April-May 2000, with the core of the line changed from a completely filled-in configuration to a moderate emission (see their Fig. 1). Although the contribution of the $\mathrm{H} \beta$ line is weaker than that of $\mathrm{H} \alpha$, it also shows evident changes. It appears as a partially filled-in absorption line during its minimum, while its core reaches the continuum level during the maximum (see Fig. 3).

We measured the radiative losses in these two lines, again following a similar process as in Frasca \& Catalano (1994), that 


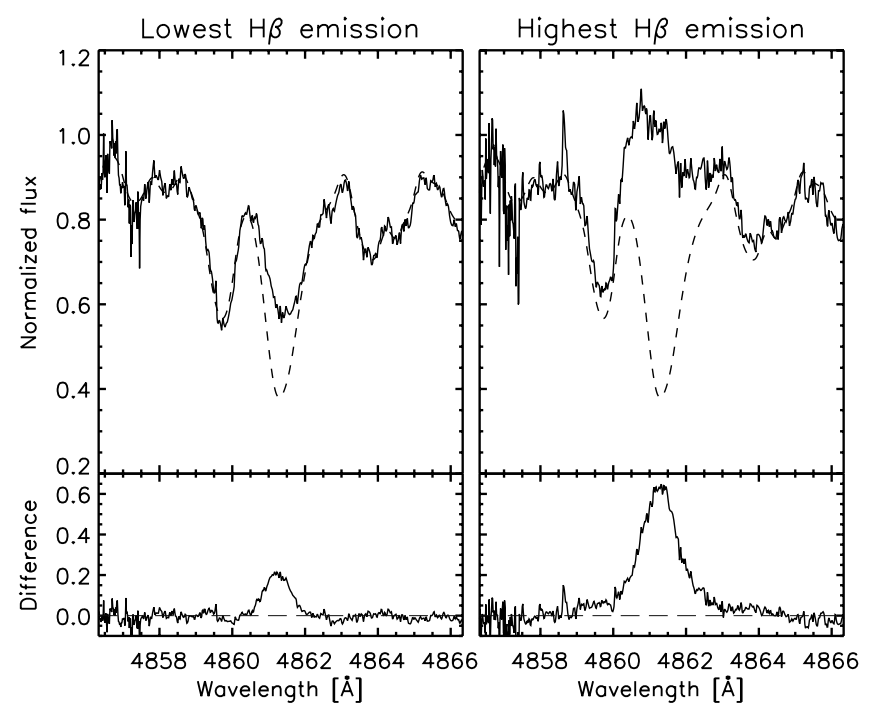

Fig. 3. As Fig. 2, but for the $\mathrm{H} \beta$ line.

is, by multiplying the equivalent width of the line by the continuum surface flux at its respective wavelength,

$F_{\mathrm{H}_{\alpha}, \mathrm{H}_{\beta}}=E W_{\mathrm{H}_{\alpha}, \mathrm{H}_{\beta}} F_{\mathrm{C}_{6563,4861}}$.

The continuum surface fluxes are estimated with the program SPECTRUM (Gray \& Corbally 1994). We used model atmospheres from Kurucz (1993) and the temperatures calculated with PARSES, a $\log g$ of 4.0 (Kővári et al. 2004), and the abundances from Rice \& Strassmeier (1998). A phase-plot of the chromospheric radiative losses of $\mathrm{H} \alpha$ is presented in the upper panel of Fig. 4. The figure shows a clear rotational modulation with lowest fluxes in the range $3-4 \times 10^{6} \mathrm{erg} \mathrm{cm}^{-2} \mathrm{~s}^{-1}$ near phase 0.0 , increasing to $4-7 \times 10^{6} \mathrm{erg} \mathrm{cm}^{-2} \mathrm{~s}^{-1}$ at phase 0.6 . When we exclude values with fluxes $\gtrsim 5 \times 10^{6} \mathrm{erg} \mathrm{cm}^{-2} \mathrm{~s}^{-1}$ from the sample, we observe that the intrinsic scatter of the measurements is $1 \times 10^{6} \mathrm{erg} \mathrm{cm}^{-2} \mathrm{~s}^{-1}$ and the rms $4 \times 10^{6} \mathrm{erg} \mathrm{cm}^{-2} \mathrm{~s}^{-1}$. The radiative losses from $\mathrm{H} \beta$ (not plotted here) show a very similar behavior that correlates well with $\mathrm{H} \alpha$ (Pearson correlation coefficient $\rho=0.95$ ).

Additionally, we evaluated the Balmer decrement $F_{\mathrm{H} \alpha} / F_{\mathrm{H} \beta}$ as a first estimate of the emission source. Dense chromospheric structures such as flares or plages have Balmer decrements typically in the range 1-3 (e.g., Zirin et al. 1982; Chester 1991), while for prominences seen off-limb, the values rise to near 10 (e.g., Landman \& Mongillo 1979). For LQ Hya, we again found a clear phase-modulation (see bottom panel in Fig. 4). The hemisphere of the star with more chromospheric emission also shows a smaller Balmer decrement ( 2.6) than the less active side ( 3.0). These values indicate that the chromospheric emission is dominated by flares and/or plages, with possibly a marginal contribution of prominences, and that either the plages are more concentrated on the active hemisphere or flares occur more frequently in this area.

\subsection{Chromospheric electron density}

We estimated the electron densities for the lower chromosphere of LQ Hya by assuming an isothermal chromosphere with $T_{\text {chrom }}=10000 \mathrm{~K}$, and the formulation of Cram \& Mullan (1979). Under these assumptions, the electron density can be evaluated as

$n_{\mathrm{e}}=1.67 \times 10^{14} \frac{F_{\text {max }}}{F_{\text {cont }}} \frac{B\left(T_{\text {eff }}\right)}{B\left(T_{\text {chrom }}\right)} \exp \left(\frac{\Delta \lambda_{\text {peak }}}{2 \Delta \lambda_{\mathrm{D}}}\right)^{-2}$,

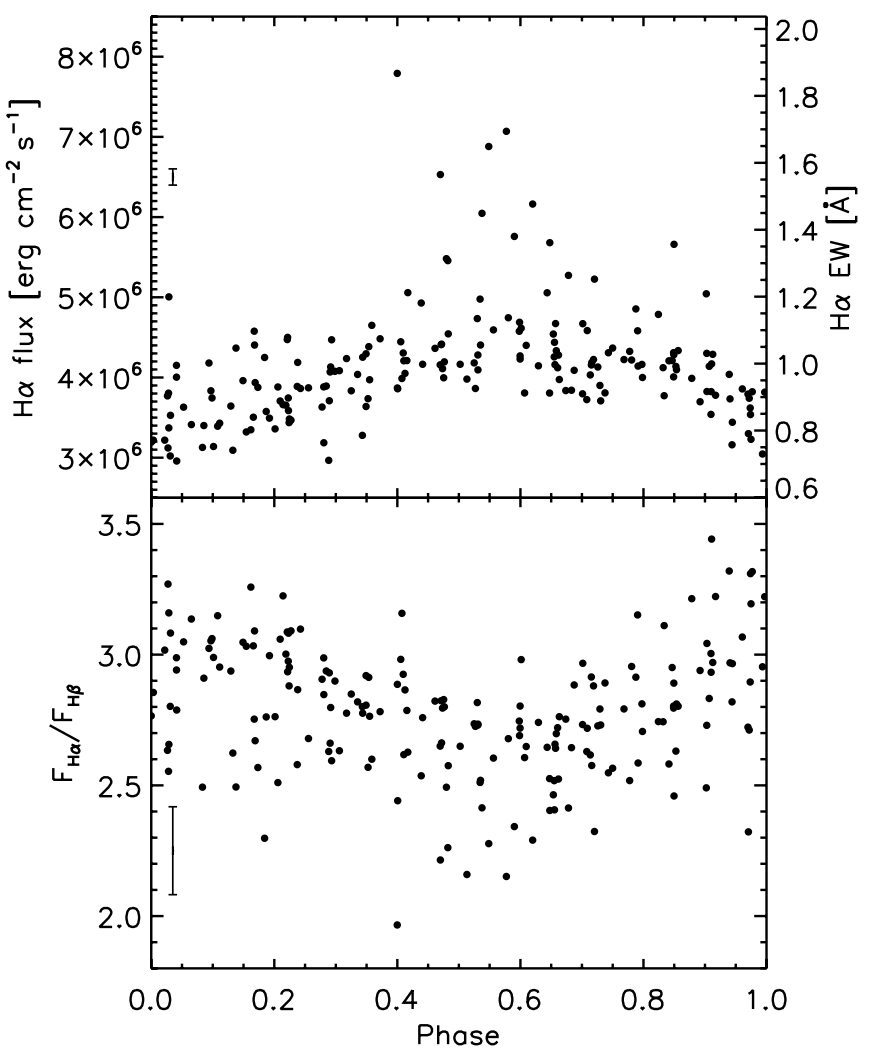

Fig. 4. Top panel: chromospheric radiative losses in $\mathrm{H} \alpha$ versus rotational phase. To facilitate the comparison with other works, the equivalent-width scale is shown at the right-hand side of the plot. Because of the individual temperatures, the two magnitudes are not exactly proportional. The differences are small, however. Measured EWs are provided in the online data. Bottom panel: phase-plot of the Balmer decrement $F_{\mathrm{H} \alpha} / F_{\mathrm{H} \beta}$. Average error bars are given at the left side of each panel.

where $F_{\max } / F_{\text {cont }}$ is the ratio of the highest $\mathrm{H} \alpha$ flux to the continuum flux, $B\left(T_{\text {eff }}\right)$ and $B\left(T_{\text {chrom }}\right)$ are the Planck functions evaluated for the temperatures calculated with PARSES and for $T_{\text {chrom }}=10000 \mathrm{~K}$ respectively, $\Delta \lambda_{\text {peak }}$ is the separation of the blue and red emission peaks, and $\Delta \lambda_{\mathrm{D}}$ is the chromospheric Doppler width of the line obtained from

$\Delta \lambda_{\mathrm{D}}=12.85\left(\frac{T_{\text {chrom }}}{10^{4} \mathrm{~A}}\right)^{1 / 2} \frac{\lambda_{0}}{c}$,

where $A=1 \mathrm{u}$ is the atomic weight, and $\lambda_{0}=6562.79 \AA$ is the rest wavelength of $\mathrm{H} \alpha$ (see Mihalas 1970). As the noise level of some $\mathrm{H} \alpha$ spectra is too high to accurately measure $\Delta \lambda_{\text {peak }}$, we decided to use ten phase-averaged profiles instead of measuring over single spectra. To calculate these profiles, we divided our spectral dataset into ten groups according to their rotational phase, each group therefore with a width of 0.1 phases, and took the weighted mean over them, using $\mathrm{S} / \mathrm{N}$ as its weighting function. We note that the interpretation of these averaged profiles is still hampered by possible intrinsic variations in magnetic activity. The errors of the electron density were evaluated by propagating the uncertainties in Eq. (2), where we have estimated $\sigma_{\Delta \lambda_{\text {peak }}}=0.25 \AA$ and $\sigma_{F_{\max } / F_{\text {cont }}}=0.01$.

Figure 5 shows that the electron density varies widely, ranging from values as low as $n_{\mathrm{e}}=(1.5 \pm 0.1) \times 10^{10} \mathrm{~cm}^{-3}$, to $n_{\mathrm{e}}=(4.4 \pm 1.3) \times 10^{12} \mathrm{~cm}^{-3}$. Furthermore, the variation is clearly rotationally modulated and correlates well with the $\mathrm{H} \alpha$ emission, pointing to a denser chromosphere above active regions. 
M. Flores Soriano et al.: Chromospheric activity and lithium line variations in the spectra of the spotted star LQ Hydrae

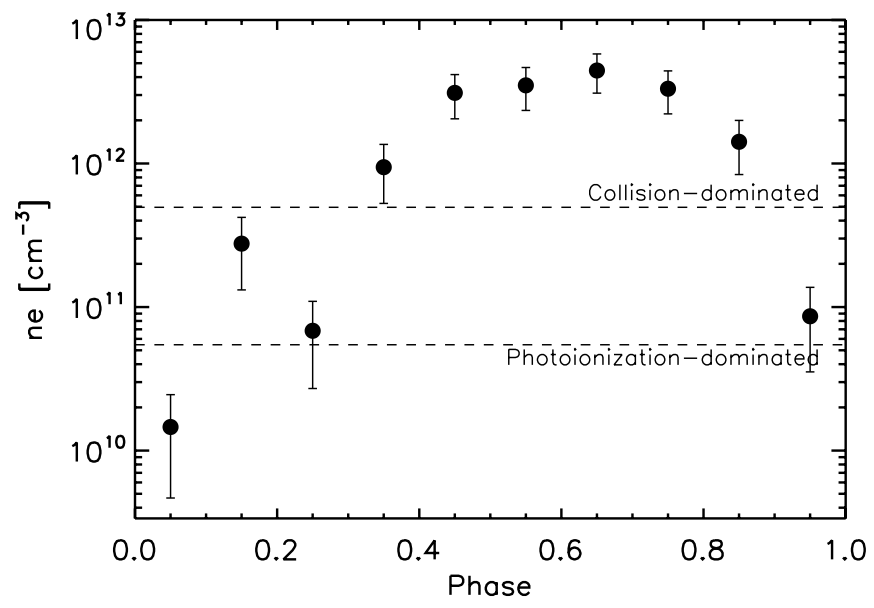

Fig. 5. Phase-plot of the chromospheric electron density measured from averaged $\mathrm{H} \alpha$ lines.

Additionally, we estimate that the source function of the $\mathrm{H} \alpha$ line is dominated by photoionization for $n_{\mathrm{e}} \lesssim 5.5 \times 10^{10} \mathrm{~cm}^{-3}$ and becomes collision-dominated for $n_{\mathrm{e}} \gtrsim 5.0 \times 10^{11} \mathrm{~cm}^{-3}$ (see Cram \& Mullan 1979, Eqs. (8) to (11)), indicating that the line formation is mainly controlled by photoionization in the less active side of the star and by collisions in the most active side. The comparison between our results and the values obtained in Papers I and II suggests that both the intensity of the magnetic activity and the homogeneity in the spatial distribution of active regions changes in time. With data from 1991, Strassmeier et al. found a homogeneously distributed electron density of $n_{\mathrm{e}} \approx 2.0 \times 10^{11} \mathrm{~cm}^{-3}$ in Paper I, while nine years later, Frasca et al. detected densities in the range $5 \times 10^{11}-4 \times 10^{12} \mathrm{~cm}^{-3}$ in Paper II, which variation was clearly modulated by the stellar rotation. We now find densities as low as $1.5 \times 10^{10} \mathrm{~cm}^{-3}$ and as high as $4.4 \times 10^{12} \mathrm{~cm}^{-3}$, which probably indicates cyclic changes.

\subsection{Sources of chromospheric emission}

Two different types of contributions can be distinguished from the $\mathrm{H} \alpha$ radiative losses (top panel of Fig. 4). The most energetic episodes (with fluxes $\gtrsim 5 \times 10^{6} \mathrm{erg} \mathrm{cm}^{-2} \mathrm{~s}^{-1}$ ) correspond to sporadic flare activity of short duration and moderate intensity (in stellar terms), occurring almost exclusively near phase 0.6. As a result of the fast rotation of the star, it is not straightforward to know the exact amount of different events, but we estimate that approximately ten of them have been detected. They show $\mathrm{H} \alpha$ profiles with broader wings (compare left and right panels of Fig. 2) that are usually asymmetric, possibly as a consequence of mass motions. (A more thorough analysis is deferred to a future work.)

The other contribution is a relatively stable and phasemodulated emission with values in the range of $3-5 \times$ $10^{6} \mathrm{erg} \mathrm{cm}^{-2} \mathrm{~s}^{-1}$. This type of regular, low-intensity activity is commonly attributed to stellar plages. Nevertheless, our estimated electron densities for the most active hemisphere of the star are at least one order of magnitude higher than the electron densities of a solar plage (e.g., Kelch \& Linsky 1978; Lemaire et al. 1981; Kucera \& Baranovsky 1994). This suggests that either the physical conditions of the active regions on LQ Hya are different from those on the Sun, or that they must be operating a more energetic process that regularly acts on that side of the star. Solar flares of importance 1+ or higher present electron densities greater than $10^{13} \mathrm{~cm}^{-3}$ (Švestka 1976 and references therein). As

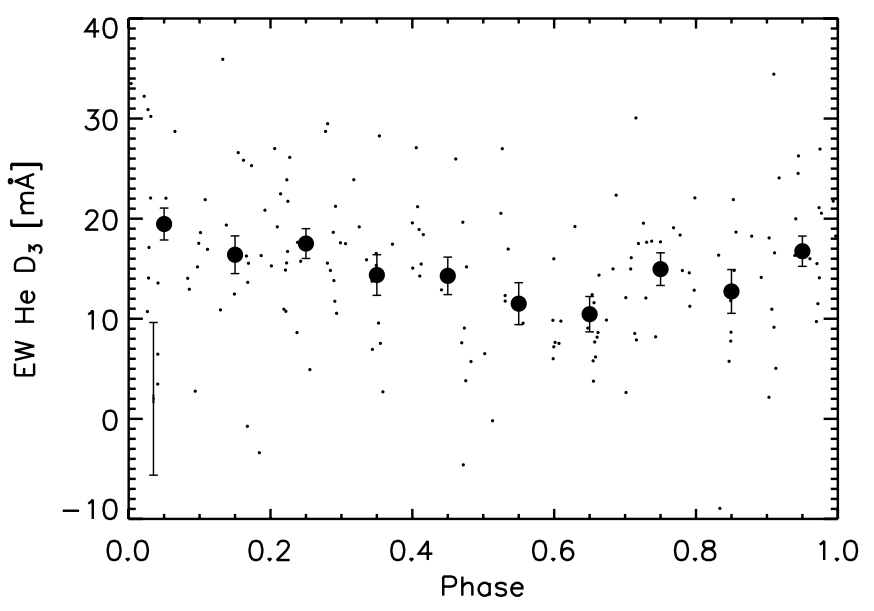

Fig. 6. Rotational modulation of the $\mathrm{He} I \mathrm{D}_{3}$ equivalent width. Measurements from single spectra are shown as small dots, while their phase-averaged values are indicated as large dots. The average error bar of single measurements is given in the lower left corner.

low-intensity flares are more common than high-intensity flares, and assuming that the physical conditions in the chromosphere of LQ Hya were approximately similar to the Sun, similar flare activity arising on a regular basis from the most active side of LQ Hya might explain the high electron densities and participate in the rotational modulation.

To test this hypothesis, we analyzed the behavior of the $\mathrm{He} \mathrm{I}_{3}$ line. It appears as an absorption feature in solar plages (Landman 1981) while it can be observed in emission in solar flares (Švestka 1976; Zirin 1988). We evaluated the equivalent width of the line for each one of the 176 spectra that are not associated with sporadic flares $\left(F_{\mathrm{H} \alpha} \leq 4.7 \times 10^{6} \mathrm{erg} \mathrm{cm}^{-2} \mathrm{~s}^{-1}\right)$ using a constant integration window of $3 \AA$. To clean the line from blends, we subtracted the non-active reference spectrum of HD 3765, but the measurements are still affected by telluric lines. The results are shown in Fig. 6. Although the value dispersion is high because of the comparably low $\mathrm{S} / \mathrm{N}$ and the weakness of the line, we observe that its strength decreases at phase 0.6 in clear anticorrelation with the magnetic activity. In this way, a higher concentration of solar-like plages on the most active side of the star can be rejected as the sole source of chromospheric emission, as it would imply the enhancement of the $\mathrm{He} \mathrm{I}_{3}$ line. It appears that this behavior is compatible with the hypothesis of low-intensity flare activity occurring regularly in that area of the star, as it would produce some filling-in of the line. Nevertheless, we note that a similar effect could be produced by a locally denser chromosphere as collisions would be more important in the $\mathrm{He} \mathrm{I}_{3}$ line formation. Considering that this local density increment would require the participation of magnetic fields and that sporadic flare activity is present, it is possible that both contributions could be taking place simultaneously. In any case, their relative importance remains unclear.

\section{Lithium I line analysis}

\subsection{Equivalent width variation}

In this section we investigate a possible connection between the rotationally modulated magnetic activity and the abundance of lithium. The most common line for $\mathrm{Li}$ abundance determinations is the resonance doublet Li I $6708 \AA$ which in cool stars with comparable broad lines appears slightly blended with Fe I and other minor lines, mostly vanadium and $\mathrm{CN}$. As a first step, 


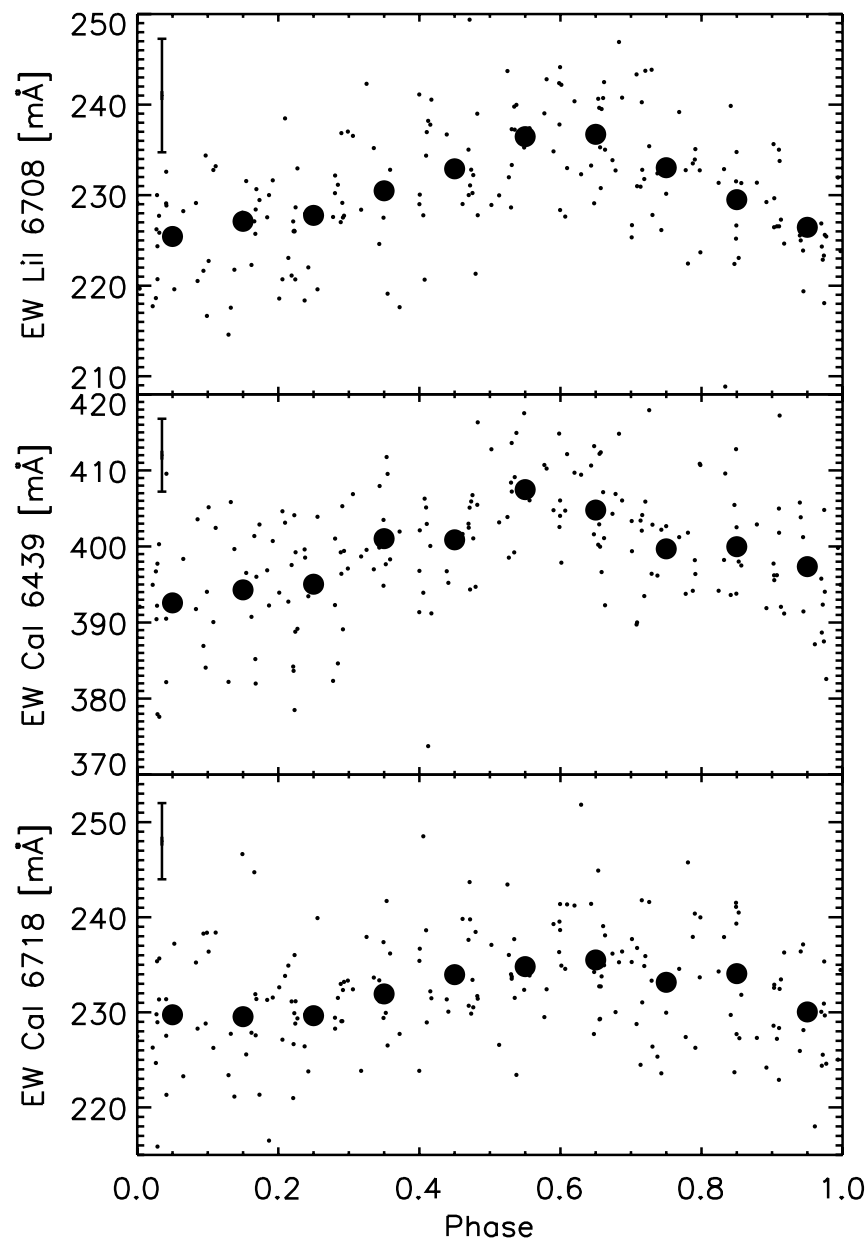

Fig. 7. Equivalent width as a function of the rotational phase for the lines LiI $6708 \AA$ (top panel), CaI $6439 \AA$ (middle), and Ca I $6718 \AA$ (bottom). Small dots correspond to measurements from single spectra, while large dots are phase-averaged values. The average error bar of single measurements is indicated in the upper left corner of each panel.

we calculated the equivalent width of the Li line for each one of the 199 LQ Hya spectra. To obtain the value free of blends, we integrated the profiles after subtracting the HD 3765 template, which does not show any Li line above our detection threshold. EW uncertainties were estimated using Eq. (1). As can be seen in the top panel of Fig. 7, the strength of the Li I $6708 \AA$ line is rotationally modulated, and its variations are well correlated with the magnetic activity. The equivalent width of the line changes from $226 \mathrm{~m} \AA$ at the phase where the lowest $\mathrm{H} \alpha$ emission has been detected, to $237 \mathrm{~m} \AA$ at the phase with highest emission. For comparison, Fekel et al. (1986a) measured an equivalent width of $234 \mathrm{~m} \AA$ for this star.

Since the ionization potential of lithium is only $5.37 \mathrm{eV}$ and the excitation potential of the line is $\chi=0.00 \mathrm{eV}$, its strength is very sensitive to temperature variations. We showed in the middle panel of Fig. 1 that the side of the star with more $\mathrm{H} \alpha$ emission is also $\sim 30 \mathrm{~K}$ cooler than the opposite side, indicating a higher concentration of cool spots. To determine wether these spots are responsible for the Li line enhancement, we evaluated the equivalent widths of two other temperature-sensitive lines (see middle and bottom panels of Fig. 7). The first line is the Ca I $6439 \AA$ line $(\chi=2.53 \mathrm{eV})$, which shows a similar rotational modulation, and is on average enhanced by $13 \mathrm{~m} \AA$ on the

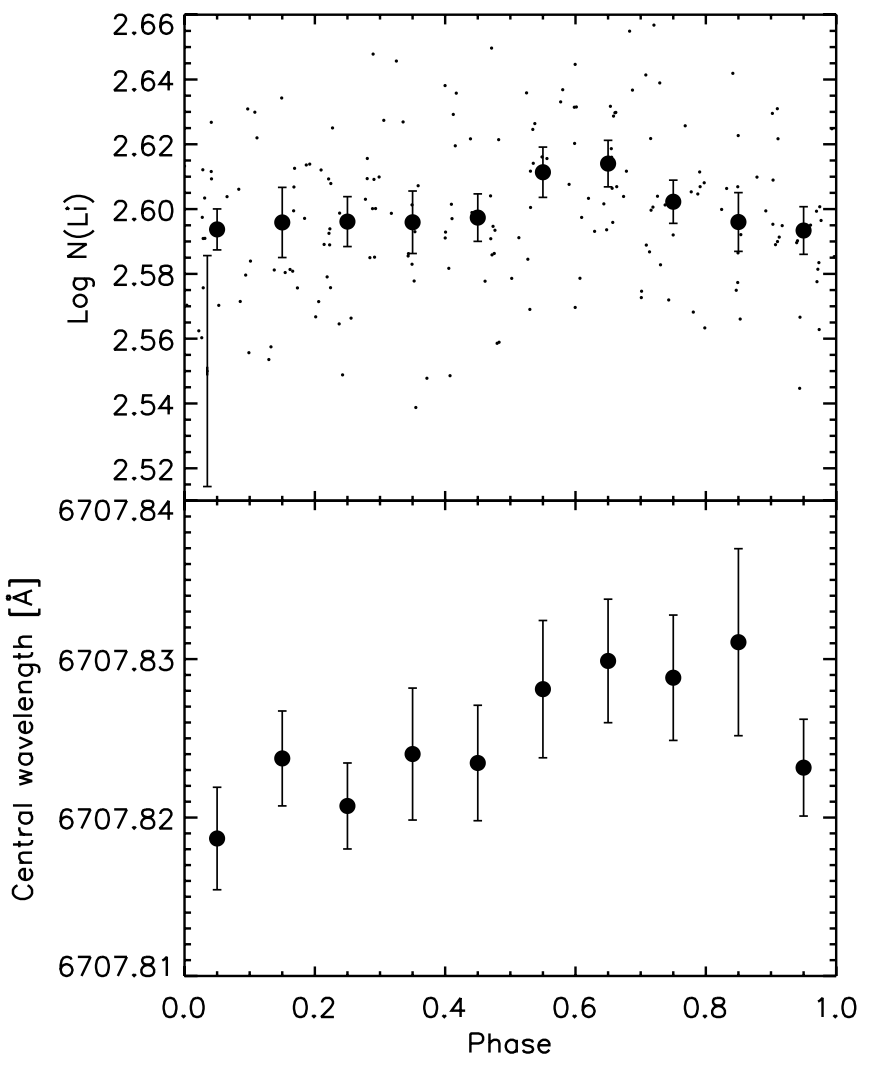

Fig. 8. Top panel: Lithium abundance from single spectra as a function of the rotational phase (small dots), and their phase-averaged values (large dots). The average error bar of single measurements is given in the lower left corner. Bottom panel: central wavelength of the (unresolved) Li I $6708 \AA$ resonance doublet. The line shift could indicate an increase of the ${ }^{6} \mathrm{Li} /{ }^{7} \mathrm{Li}$ isotopic ratio for the flaring hemisphere of the star (see text for more details).

most spotted side of the star. The second line is the neighboring Ca I $6718 \AA(\chi=2.71 \mathrm{eV})$. It is only $10 \AA$ away from the Li line and has a similar size. In this case, the enhancement is not as pronounced as for the previous lines, and the peak to peak variation is only $6 \mathrm{~m} \AA$. All this suggests that the prime source of enhancement in the lithium line could be the inhomogeneous distribution of cool spots.

\subsection{Lithium abundance}

Although an inhomogeneous distribution of $\mathrm{Li}$ in the photosphere of LQ Hya can be rejected as the primary source of variability in the Li I $6708 \AA$ line, we investigate here a possible local overabundance of this element resulting from nuclear reactions during flares. We evaluated the logarithmic lithium abundances for each one of the 199 STELLA spectra with the non-LTE curves of growth from Pavlenko \& Magazzu (1996), using the equivalent widths of the Li I $6708 \AA$ line and the temperatures and gravities from PARSES. In this way, the temperature variations are taken into account when determining the abundances, while the problems associated with the high sensitivity of the line to thermal fluctuations are mitigated. The errors of the Li abundances were estimated such that they include all values compatible with the uncertainties in equivalent width, temperature, and gravity.

In comparison with what we observed for the equivalent width, the lithium abundance shows a more stable behavior (top panel of Fig. 8). The average of the measurements gives 
a mean abundance of $\log N(\mathrm{Li})=2.6$, with a dispersion of only $\sigma=0.02$. In comparison, Fekel et al. (1986a) obtained a slightly higher value of $\log N(\mathrm{Li})=2.8$ using the curves of growth of Duncan \& Jones (1983). A more interesting behavior is revealed by taking phase-average values. For phases between 0.85 and 0.45 (i.e., for most of the stellar surface) the abundance remains remarkably constant between 2.59 and 2.60, but it increases to 2.61 at phase 0.6 , where we found the strongest chromospheric emissions. To test the statistical significance of this variation, we divided the abundances into those measured between phases 0.5 and 0.7 and those measured outside of this range. We then applied a $t$-test to compare the differences between these two samples relative to their dispersion. We found a significance of $8 \times 10^{-5}$, which indicates that it is highly improbable that the difference of the means is the result of a random fluctuation. Although the small amplitude of the variations can contrast with its high statistical significance, we note that it is a consequence of the high number of data and their relatively low local dispersion.

A limitation of the previous abundance analysis, as pointed out by our referee, is the use of equivalent widths and temperatures averaged over the visible stellar hemisphere. To test whether this approximation affects the rotational variation of the lithium abundance, we repeated our analysis using synthetic spectra. To calculate them, we first computed a simple model of the spot distribution that reproduces the rotational modulation of the temperature. The model represents the stellar surface as a grid of $60 \times 30$ pixels where the quiet photosphere and the spots have a temperature of $5000 \mathrm{~K}$ and $4780 \mathrm{~K}$. Two circular spots at latitude $30^{\circ}$, one at phase 0.52 with a radius of $18^{\circ}$, and the other at phase 0.72 with radius $12^{\circ}$ are enough to reproduce the temperature modulation. Nevertheless, this is an ill-posed problem and other spot configurations can fit the variations as well. We then computed synthetic spectra at different rotational phases with this model and the program SPECTRUM (see Sect. 3.1), using a constant lithium abundance of $\log N(\mathrm{Li})=2.6$. The analysis shows a clear variation in the equivalent width where values range from $223 \mathrm{~m} \AA$ on the hottest side to $231 \mathrm{~m} \AA$ on the coolest side. Nonetheless, the amplitude of this modulation is smaller than in the observed spectra and does not translate into any significant change in the lithium abundance. It has an average value of $\log N(\mathrm{Li})=2.59$, with a variation of 0.001 between the opposite hemispheres, and is poorly correlated with the temperature. We conclude that the use of equivalent widths and temperatures averaged over the visible hemisphere is unlikely to be the source of the observed variation.

\subsection{Variation of the ${ }^{6} \mathrm{Li} /{ }^{7} \mathrm{Li}$ isotopic ratio}

A number of authors have proposed flare nucleosynthesis models predicting amounts of ${ }^{6} \mathrm{Li}$ produced by nuclear reactions that could be high enough to increase the ${ }^{6} \mathrm{Li} /{ }^{7} \mathrm{Li}$ isotopic ratio (see, e.g., Canal et al. 1975; Walker et al. 1985; Ramaty et al. 2000; Tatischeff \& Thibaud 2007). Such an increment has previously been reported by Montes \& Ramsey (1998) during a long-duration flare in the chromospherically active binary 2RE J0743+224. Because of the relatively large isotope shift of the $\mathrm{LiI} 6708 \AA$ line, where ${ }^{6} \mathrm{Li}$ lies $0.158 \AA$ redward of ${ }^{7} \mathrm{Li}$, it could be possible, in principle, to detect a change of the ${ }^{6} \mathrm{Li} /{ }^{7} \mathrm{Li}$ isotopic ratio as a shift of the barycenter of the Li I blend toward longer wavelengths.

Because the effect is so weak, we limit our analysis to the spectra with $S / N \geq 60$. Additionally, it is important to remark
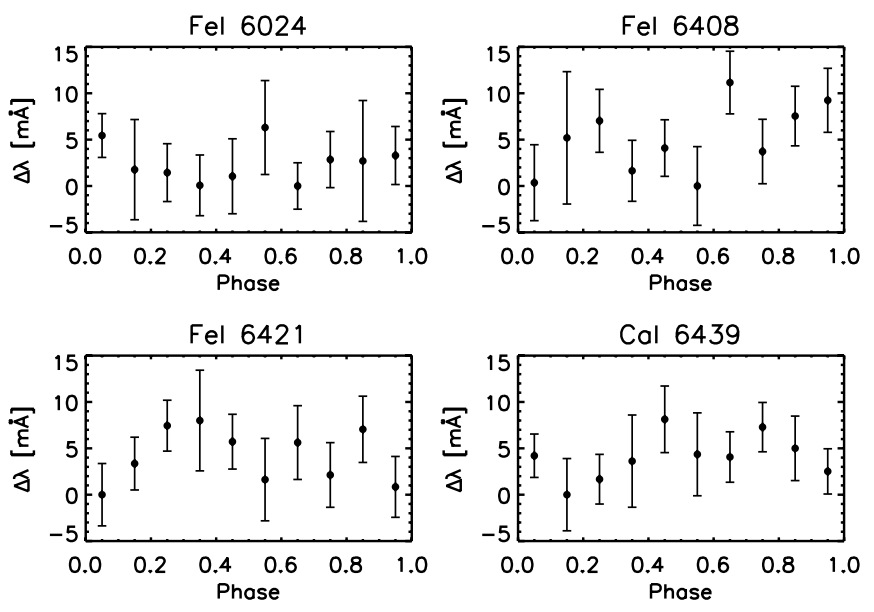

Fig. 9. Measured line-shifts for four comparison lines. The zero point corresponds to the shortest wavelength. These values were calculated to verify the variations observed in the Li I $6708 \AA$ line (bottom panel of Fig. 8). They were evaluated by measuring the position of the central wavelength relative to four reference lines (see text for details).

that chemical inhomogeneities are not the only source of line shifts. Spotted stars such as LQ Hya present bumps that travel through their spectral lines as the rotation carries the spots across the visible side of the star. To minimize the effects of these bumps, we measured the position of the Li I $6708 \AA$ line relative to four reference lines (Fe I 6393.612 A; Fe I 6411.649 Fe I $6430.856 \AA$; Ca I $6717.688 \AA$ ) that were selected for their similar strength and because they were fairly free of blends. The measurements were subsequently phase-averaged and the error bars adopted as the standard error (bottom panel of Fig. 8). According to these results, the difference between the central wavelength from opposite hemispheres leads to a redshift of Li I of $\sim 10 \pm 7 \mathrm{~m} \AA$ for the part of the star where the strongest magnetic activity has been detected. To verify this, we repeated the same analysis for other lines (Fig. 9). For these we did not find any systematic variation above the uncertainties, nor any correlation with the magnetic activity.

We tentatively converted the lithium line shifts into ${ }^{6} \mathrm{Li} /{ }^{7} \mathrm{Li}$ isotopic ratios using the approximation by Herbig (1964). According to this method, the apparent isotopic ratio can be calculated with the equation

$\frac{{ }^{6} \mathrm{Li}}{{ }^{7} \mathrm{Li}}=\frac{\lambda_{\mathrm{obs}}-6707.811}{6707.971-\lambda_{\mathrm{obs}}}$

where $\lambda_{\text {obs }}$ are the measured central wavelengths, and 6707.811 and 6707.971 are the wavelengths of the individual components, that is, for pure ${ }^{7} \mathrm{Li}$ and pure ${ }^{6} \mathrm{Li}$. This shows an increment in the isotopic ratio from $0.08 \pm 0.02$ to $0.14 \pm 0.03$. We tested the consistence between this result and the increment in the lithium abundance by assuming that the isotopic ratio on the side of the star with the lowest magnetic activity is equal to 0.08 and that the increment in the abundance is produce by new ${ }^{6} \mathrm{Li}$ atoms. Under these assumptions, the isotopic ratio should increase from the original value of 0.08 to 0.13 , which is very close to the observed value of $0.14 \pm 0.03$

\subsection{Lithium I 6104 Å line}

The only additional lithium line available in the covered spectral range is the weak and strongly blended Li I $6104 \AA$ line. 


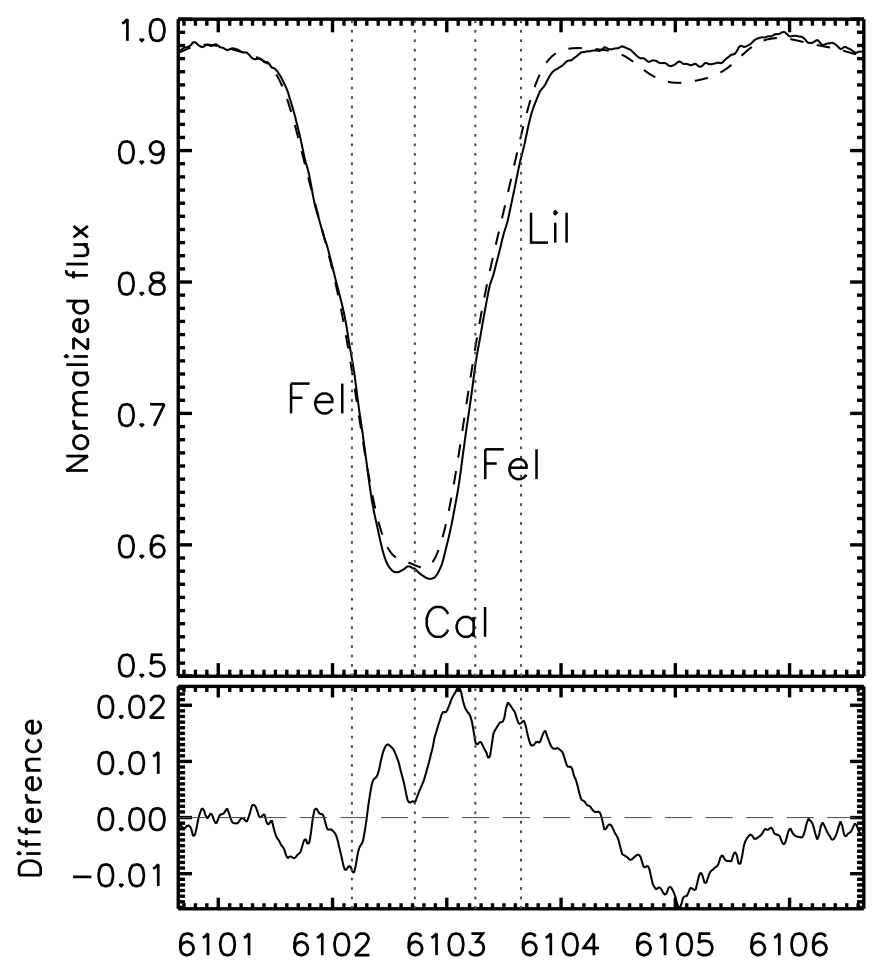

Fig. 10. Upper panel: average LQ Hya spectrum (solid) in the region around the Li I $6104 \AA$ line together with the spectrum of the reference HD 3765 star (dashed) for which we do not observe any lithium line. Bottom panel: difference between the average LQ Hya spectrum and the reference-star spectrum.

In the spectra of LQ Hya the region between 6102-6104 $\AA$ is completely dominated by a $\mathrm{Ca}$ I line and two Fe I lines. Only after subtracting the template is it possible to detect some extra absorption in the red wing of the $\mathrm{Ca}$ I-Fe I blend. As can be seen in Fig. 10, the template is unable to completely remove the contribution of the blends, and the difference profile is still contaminated. To estimate the equivalent width of the Li I $6104 \AA$ line, we used two different integration windows of 0.7 and $1.0 \AA$ that provide the lower and upper limit. Then we calculated the average of these two values and used the difference as the uncertainty. The lithium abundance was subsequently estimated with the non-LTE curves of growth from Pavlenko \& Magazzu (1996). As a result of the weakness of the line, measurements from single spectra are too scattered to study the rotational modulation. We report here an average equivalent with of $W=12 \pm 2 \mathrm{~m} \AA$, which translates into a lithium abundance of $\log N(\mathrm{Li})=2.6 \pm 0.1$, in good agreement with the $6708 \AA$ line.

\section{Summary and conclusions}

We presented and analyzed a time series of 199 moderately high resolution spectra of the young active K2 V star LQ Hya with the aim of studying the connection between magnetic activity indicators and the variability of the Li I $6708 \AA$ line.

We computed effective temperatures for each one of the STELLA spectra with the tool PARSES and found a clear rotational modulation with an amplitude of $\sim 30 \mathrm{~K}$. Quasisimultaneous $V$-band photometry shows excellent correlation and a peak-to-peak variability of $0.15 \mathrm{mag}$, indicating a higher concentration of cool spots on one side of the star. We also quantified the chromospheric activity of LQ Hya at different rotational phases from the $\mathrm{H} \alpha$ and $\mathrm{H} \beta$ lines. We found higher chromospheric emission when the most spotted side of the star is in view. In addition, low Balmer decrements $F_{\mathrm{H} \alpha} / F_{\mathrm{H} \beta}$, in combination with high electron densities and the modulation of the $\mathrm{He}$ I $\mathrm{D}_{3}$ line suggest that the emission is mainly due to chromospheric features with physical conditions similar to strong solar flares. Sporadic flares of moderate intensity were also detected.

Taking advantage of the spatially inhomogeneous distribution of spots and flare activity, we studied the effect of cool spots on the strength of the Li I $6708 \AA$ line and whether new Li has been produced by nuclear reactions during flares. We observed that the strength of the Li line and other temperature sensitive lines is rotationally modulated and clearly enhanced in the hemisphere of the star with the highest magnetic activity, but this is mainly an effect of the modulation of the average temperature. We also calculated the lithium abundance for each one of the 199 STELLA spectra taking into account the hemispheric temperature variations. Although it exhibits a much lower rotational amplitude, it is still possible to detect a small but statistically significant overabundance ( $t$-test significance of $\left.8 \times 10^{-5}\right)$ in the hemisphere with the highest activity. Simulations made with synthetic spectra by combining spotted regions and immaculate photosphere were used to determine whether the lithium EW and abundance variations are only owe to the modulation of the effective temperature or include an intrinsic origin. They show that the source of the observed lithium enhancement is probably not the use of hemispheric average equivalent widths and temperatures to compute the abundances. We also analyzed the Li I $6708 \AA$ line shifts as an indicator for the production of ${ }^{6} \mathrm{Li}$ by flares. We found that the line is red-shifted by $\sim 10 \pm 7 \mathrm{~m} \AA$ when the most active side of the star is in view. None of the other lines that we tested shows such a clear variation with this amplitude.

Even though starspots are the prime source of variability in the LiI $6708 \AA$ line, our finding of a rotationally modulated $\mathrm{Li}$ abundance and a systematic redshift of the line center at higher activity seemingly supports the hypothesis that fresh lithium is produced by flares. We have shown that the magnitudes of these two increments agree well with each other in a scenario where the new lithium atoms were mostly ${ }^{6} \mathrm{Li}$. Moreover, their good correlation with the rotational modulation of other activity indicators such as the V-light curve, $\mathrm{H} \alpha$ and $\mathrm{H} \beta$ emission, and $\mathrm{He} \mathrm{I}_{3}$ variations strongly supports a relation with the magnetic activity. Because this effect is weak and the dispersion in the measurements from single spectra is high we cannot consider any of these two factors as entirely conclusive by itself, but the good correlation between different variables and the fact that the variations of phase-averaged values are in both cases higher than the estimated uncertainties seems to disprove the possibility of them being just a random fluctuation.

As a result of the high sensitivity of the line to temperature, any hypothetical underestimation of the temperature fluctuation by our PARSES solution would translate into an even smaller variation in the Li abundance, but it would have no effect on the line shifts. It is also known that the region near the Li line is affected by a number of very weak lines whose nature and properties are still a matter of research (see, for example, Mandell et al. 2004) and by the hyperfine structure of Li itself. The effects of these lines on our measurements are thus uncertain. On the other hand, differential rotation acting during the four months of observations could blur the measurements and reduce the significance of the variations. For these reasons, considering that none of the other causes of variability seems to be a better alternative, we suggest that a local overabundance of lithium could exist on the side of LQ Hya with the strongest magnetic activity. 
M. Flores Soriano et al.: Chromospheric activity and lithium line variations in the spectra of the spotted star LQ Hydrae

Acknowledgements. We thank an anonymous referee, whose comments and suggestions improved the paper. We are grateful to the State of Brandenburg and the German federal ministry for education and research (BMBF) for their continuous support of the STELLA and APT activities. The STELLA facility is a collaboration of the AIP in Brandenburg with the IAC in Tenerife. It is our pleasure to thank Lou Boyd for nursing the APTs at Fairborn Observatory.

\section{References}

Allende Prieto, C. 2004, Astron. Nachr., 325, 604

Allende Prieto, C., Beers, T. C., Wilhelm, R., et al. 2006, ApJ, 636, 804

Ambruster, C., \& Fekel, F. 1990, in BAAS, 22, 857

Bidelman, W. P. 1981, AJ, 86, 553

Brown, J. A., Sneden, C., Lambert, D. L., \& Dutchover, Jr., E. 1989, ApJS, 71, 293

Canal, R. 1974, ApJ, 189, 531

Canal, R., Isern, J., \& Sanahuja, B. 1975, ApJ, 200, 646

Cao, D.-T., \& Gu, S.-H. 2014, AJ, 147, 38

Chester, M. M. 1991, Ph.D. Thesis, Pennsylvania State University, University Park

Covino, S., Panzera, M. R., Tagliaferri, G., \& Pallavicini, R. 2001, A\&A, 371, 973

Cram, L. E., \& Mullan, D. J. 1979, ApJ, 234, 579

Cutispoto, G. 2002, Astron. Nachr., 323, 325

Deliyannis, C. P., \& Malaney, R. A. 1995, ApJ, 453, 810

Duncan, D. K., \& Jones, B. F. 1983, ApJ, 271, 663

Favata, F., Micela, G., \& Sciortino, S. 1996, A\&A, 311, 951

Fekel, F. C. 1996, in Stellar Surface Structure, eds. K. G. Strassmeier, \& J. L. Linsky (Kluwer Academic Press), IAU Symp., 176, 345

Fekel, F. C., Bopp, B. W., Africano, J. L., et al. 1986a, AJ, 92, 1150

Fekel, F. C., Moffett, T. J., \& Henry, G. W. 1986b, ApJS, 60, 551

Fowler, W. A., Burbidge, G. R., \& Burbidge, E. M. 1955, ApJS, 2, 167

Frasca, A., \& Catalano, S. 1994, A\&A, 284, 883

Frasca, A., Kővári, Z., Strassmeier, K. G., \& Biazzo, K. 2008, A\&A, 481, 229 (Paper II)

Frasca, A., Fröhlich, H.-E., Bonanno, A., et al. 2011, A\&A, 532, A81

Giampapa, M. S. 1984, ApJ, 277, 235

Granzer, T., Reegen, P., \& Strassmeier, K. G. 2001, Astron. Nachr., 322, 325

Gray, R. O., \& Corbally, C. J. 1994, AJ, 107, 742

Grevesse, N. 1968, Sol. Phys., 5, 159

Gustafsson, B., Edvardsson, B., Eriksson, K., et al. 2008, A\&A, 486, 951

Heintz, W. D. 1981, ApJS, 46, 247

Herbig, G. H. 1964, ApJ, 140, 702

Herbig, G. H. 1985, ApJ, 289, 269

Iben, Jr., I. 1967, ApJ, 147, 624

Jovanovic, M., Weber, M., \& Allende Prieto, C. 2013, Publications de l'Observatoire Astronomique de Beograd, 92, 169

Kelch, W. L., \& Linsky, J. L. 1978, Sol. Phys., 58, 37

Kővári, Z., Strassmeier, K. G., Granzer, T., et al. 2004, A\&A, 417, 1047
Kucera, A., \& Baranovsky, E. A. 1994, in Infrared Solar Physics, eds. D. M. Rabin, J. T. Jefferies, \& C. Lindsey (Kluwer Academic Publishers), IAU Symp., 154, 29

Kupka, F., Dubernet, M.-L., \& VAMDC Collaboration 2011, Balt. Astron., 20, 503

Kurucz, R. 1993, ATLAS9 Stellar Atmosphere Programs and $2 \mathrm{~km} \mathrm{~s}^{-1}$ grid. Kurucz CD-ROM No. 13 (Cambridge, Mass.: Smithsonian Astrophysical Observatory)

Landman, D. A. 1981, ApJ, 244, 345

Landman, D. A., \& Mongillo, M. 1979, ApJ, 230, 581

Lemaire, P., Gouttebroze, P., Vial, J. C., \& Artzner, G. E. 1981, A\&A, 103, 160

Li, T. D., Bi, S. L., Yang, W. M., et al. 2014, ApJ, 781, 62

Mandell, A. M., Ge, J., \& Murray, N. 2004, AJ, 127, 1147

Mathioudakis, M., Drake, J. J., Craig, N., et al. 1995, A\&A, 302, 422

Mihalas, D. 1970, Stellar atmospheres (San Francisco: Freeman)

Montes, D., \& Ramsey, L. W. 1998, A\&A, 340, L5

Montes, D., Saar, S. H., Collier Cameron, A., \& Unruh, Y. C. 1999, MNRAS, 305,45

Pallavicini, R., Randich, S., \& Giampapa, M. S. 1992, A\&A, 253, 185

Pavlenko, Y. V., \& Magazzu, A. 1996, A\&A, 311, 961

Prugniel, P., \& Soubiran, C. 2001, A\&A, 369, 1048

Ramaty, R., Tatischeff, V., Thibaud, J. P., Kozlovsky, B., \& Mandzhavidze, N. 2000, ApJ, 534, L207

Rice, J. B., \& Strassmeier, K. G. 1998, A\&A, 336, 972

Saar, S. H., \& Bookbinder, J. A. 1998, in Cool Stars, Stellar Systems, and the Sun, eds. R. A. Donahue, \& J. A. Bookbinder, ASP Conf. Ser., 154, 1560

Soderblom, D. R., Jones, B. F., Balachandran, S., et al. 1993, AJ, 106, 1059

Strassmeier, K. G., Rice, J. B., Wehlau, W. H., Hill, G. M., \& Matthews, J. M. 1993, A\&A, 268, 671 (Paper I)

Strassmeier, K. G., Boyd, L. J., Epand, D. H., \& Granzer, T. 1997, PASP, 109, 697

Strassmeier, K. G., Granzer, T., Weber, M., et al. 2010, Adv. Astron., 2010, 970306

Strassmeier, K. G., Weber, M., Granzer, T., \& Järvinen, S. 2012, Astron. Nachr., 333,663

Strassmeier, K. G., Carroll, T. A., Weber, M., \& Granzer, T. 2015, A\&A, 574, A31

Švestka, Z. 1976, Solar Flares (Berlin, Heidelberg: Springer-Verlag)

Tatischeff, V., \& Thibaud, J.-P. 2007, A\&A, 469, 265

Traub, W., \& Roesler, F. L. 1971, ApJ, 163, 629

Ventura, P., Zeppieri, A., Mazzitelli, I., \& D’Antona, F. 1998, A\&A, 331, 1011

Walker, T. P., Viola, V. E., \& Mathews, G. J. 1985, ApJ, 299, 745

Wallerstein, G., \& Sneden, C. 1982, ApJ, 255, 577

Weber, M., \& Strassmeier, K. G. 2011, A\&A, 531, A89

Weber, M., Granzer, T., Strassmeier, K. G., \& Woche, M. 2008, in SPIE Conf. Ser., 7019

Zirin, H. 1988, Astrophysics of the Sun (Cambridge and New York: Cambridge University Press)

Zirin, H., Liggett, M., \& Patterson, A. 1982, Sol. Phys., 76, 387 